\title{
Uma análise do emprego do Método Multigrid Algébrico na simulação numérica da propagação de ondas oceânicas
}

\section{An analysis of the use of the Algebraic Multigrid Method in the numerical simulation of the propagation of ocean waves}

Roberta Suero ${ }^{1}$, Nielton de Souza Monteiro²,Paulo Ricardo Ferreira de Miranda ${ }^{3}$, Matheus José de Deus ${ }^{4}$, Elizaldo Domingues dos Santos ${ }^{5}$, Liércio André Isoldi ${ }^{6}$, Luiz Alberto Oliveira Rocha7, Mateus das Neves Gomes ${ }^{8}$

\section{RESUMO}

O presente trabalho apresenta uma análise do emprego do Método Multigrid Algébrico (AMG) nas simulações numéricas, que envolvem a propagação de ondas oceânicas em tanques numéricos. O método multigrid é um dos métodos mais efetivos para acelerar a convergência de métodos iterativos e resolver sistemas lineares ou não lineares. São realizadas três diferentes análises com o objetivo de diminuir o tempo de processamento das simulações numéricas da propagação e ondas: i) número de pré e pós suavização, ii) número de níveis e iii) número de passos de suavização. Para as análises do método multigrid é considerada a propagação de uma onda com período de $5 \mathrm{~s}$ e comprimento de $37,5 \mathrm{~m}$. O domínio computacional é bidimensional e representado por um tanque de ondas numérico dividido em uma região de propagação e outra de absorção da onda. Para a solução numérica foi empregado um código de dinâmica dos fluidos computacional, FLUENT ${ }^{\circledR}$ baseado no Método de Volumes Finitos (MVF). O modelo multifásico Volume of Fluid (VOF) é aplicado no tratamento da interação água-ar. Foram testados diferentes valores do número de pré e pós suavização, do número de níveis e do número de passos de suavização e os resultados conduziram a uma redução de tempo de 10,16 h.

Palavras-chave: Multigrid Algébrico, Ondas, Tanques, VOF, Tempo de Processamento.

\section{ABSTRACT}

The present work presents an analysis of the use of the Algebra Multigrid Method (AMG) in numerical simulations, which involve the propagation of ocean waves in numerical tanks. The multigrid method is one of the most effective methods to accelerate the convergence of iterative methods and to solve linear or nonlinear systems. Three different analyzes are performed with the purpose of reducing the computational time of the propagation and wave number simulations: i) number of pre and post smoothing, ii) number of levels and iii) number of smoothing steps. For the analyzes of the multigrid method the propagation of a wave with period of $5 \mathrm{~s}$ and length of $37.5 \mathrm{~m}$ is considered. The computational domain is two-dimensional and represented by a numerical wave pool divided into one region of propagation and one of wave absorption. For the numerical solution, a computational fluid dynamics code, FLUENT®, was used based on the Finite Volume Method (MVF). The Multiphase Volume of Fluid (VOF) model is applied in the treatment of water-air interaction. Different values of the number of pre and post smoothing, the number of levels and the number of smoothing steps were tested and the results led to a time reduction of $10,16 \mathrm{~h}$

Keywords: Algebraic Multigrid, Waves, Tanks, VOF, Processing Time.
${ }^{1}$ Doutora em Métodos Numéricos

em Engenharia, IFPR

roberta.suero@ifpr.edu.br

${ }^{2}$ Graduando em Tecnologia em

Manutenção Industrial, IFPR,

nieltonmonteiro3@hotmail.com.

${ }^{3}$ Graduando em Tecnologia em

Manutenção Industrial, IFPR,

paulo_miranda14@hotmail.com.

${ }^{4}$ Graduando em Licenciatura em

Física, IFPR,

matheus.pgua@hotmail.com.

${ }^{5}$ Doutor em Engenharia Mecânica,

FURG, elizaldosantos@furg.br.

${ }^{6}$ Doutor em Engenharia Mecânica,

FUGS, liercioisoldi@furg.br.

${ }^{7}$ Doutor em Engenharia Mecânica UNISINOS, luizor@unisinos.br.

${ }^{8}$ Doutor em Engenharia Mecânica, IFPR, mateus.gomes@ifpr.edu.br 


\section{INTRODUÇAOO}

Em engenharia, existem três tipos de abordagens para o desenvolvimento de projetos e análises de problemas: métodos analíticos; métodos numéricos (experimentação numérica); e experimentação física em laboratório (MALISKA, 2004).

Desde longa data, trabalhos experimentais de Engenharia Oceânica e Arquitetura Naval têm usado tanques de ondas (CARNEIRO, 2007). Através deste tipo de abordagem é possível reproduzir condições de mar de forma simplificada em laboratórios devidamente equipados. Desta forma, diversas estruturas oceânicas podem ser analisadas, como por exemplo: embarcações, plataformas de petróleo, dispositivos conversores de energia das ondas do mar entre outros equipamentos.

Outra abordagem, complementar a experimentação física, é através de experimentos numéricos. Uma forma de representação computacional do fenômeno de propagação de ondas é através de tanques numéricos de ondas. Diversos trabalhos que são encontrados na literatura, como por exemplo: Gomes et al. (2009), Gomes (2014), Martins et al. (2018), empregam este tipo de abordagem através do método Volume Of Fluid (VOF), proposto por Hirt e Nichols (1981). Esse método é recomendado para escoamentos multifásicos onde há dois ou mais fluidos com uma interface muito bem definida, que no presente estudo são o ar e a água. Versteeg e Malalasekera (2007) propõe a solução das equações deste modelo matemático através do Método dos Volumes Finitos (MVF).

Em simulação numérica um importante aspecto a ser considerado é o tempo de processamento, que depende de diversos fatores como, por exemplo, a discretização espacial e temporal. Esta discretização conduz a representação do problema através de sistemas de equações, em muitos casos com milhares de incógnitas. Uma forma de acelerar a convergência dos métodos iterativos de resolução de sistemas é o método multigrid, que pode ser empregado conforme duas abordagens: método multigrid geométrico (GMG) e método multigrid algébrico (AMG). De acordo com Trottenberg et al. (2001), o método multigrid é caracterizado por suas componentes: procedimentos de suavização (solvers), estratégia de engrossamento, operador de malha grossa, operadores de transferência de malhas finas para malhas grossas (e vice-versa) e tipo de ciclo. 
$\mathrm{Na}$ literatura podem ser encontrados diversos trabalhos sobre o estudo das componentes do Método Multigrid. Oliveira et al. (2018), estudam os efeitos dos parâmetros do método multigrid geométrico em uma equação da Difusão de Calor 3D. Concluem que, tanto o número de suavizações, quanto o número de níveis influenciam no tempo de processamento. Rutz e Pinto (2016) discutem, dentre outros fatores, os mesmos parâmetros, concluindo que, os mesmos, influenciam diretamente o tempo computacional gasto na resolução do problema, que trata da equação da difusão anisotrópica. Santiago et al. (2015) resolve as equações bidimensionais de Laplace, Navier, Burgers e duas formulações de Navier-Stokes. Eles demonstram que, o número de iterações internas e o número de níveis empregados para a resolução via método multigrid geométrico, influenciam no tempo computacional. Suero et al. (2012), resolvem a equação da difusão, empregando malhas quadrangulares e triangulares, com o uso do Método Multigrid Algébrico. Neste trabalho também é comprovado que o número de iterações internas e o número de níveis influenciam no tempo computacional gasto na resolução do problema.

Desta forma, o objetivo deste trabalho é analisar o emprego do Método Multigrid Algébrico nas simulações numéricas, que envolvem a propagação de ondas oceânicas em tanques. São testados diferentes valores para o número de iterações internas e o número de níveis com o objetivo de acelerar a convergência e consequentemente reduzir o tempo de processamento das simulações numéricas supracitadas. Para tanto, é utilizado um código de volumes finitos disponível no software ANSYS FLUENT.

\section{MATERIAIS E METODOS}

\subsection{MÉTODO MULTIGRID ALGÉBRICO}

O método multigrid é um dos métodos mais efetivos para acelerar a convergência de métodos iterativos e resolver sistemas lineares ou não lineares, problemas anisotrópicos, dentre outros (BRIGGS et al., 2000; TROTTENBERG et al., 2001; WESSELING et al., 2001). Este método proporciona taxa de convergência independente do tamanho da malha e é muito eficaz para resolver problemas que exigem grande esforço computacional (GUPTA et al., 1997). De acordo com Stüben (2001), a eficiência do método multigrid não tem sido totalmente alcançada em aplicações realísticas de CFD. 
Conforme Wesseling (1992), o método multigrid consiste no uso de malhas auxiliares mais grossas (com menor número de nós) do que a malha na qual se quer resolver o problema. Para transferir as informações da malha fina para a malha imediatamente mais grossa (restrição) e da malha grossa para a malha imediatamente mais fina (prolongação) são utilizados operadores de transferência. A seqüência com que as diversas malhas são visitadas caracteriza um ciclo multigrid, que pode ser do tipo $\mathrm{V}, \mathrm{W}$ e F, entre outros (BRIGGS et al., 2000; TROTTENBERG et al., 2001). Os sistemas lineares, em cada malha, são resolvidos com um método iterativo (solver) com propriedades de reduzir rapidamente os erros oscilatórios (propriedades de suavização). Podem ser usados dois tipos de esquemas (BRIGGS et al., 2000): o esquema de correção (Correction Scheme, CS) e o esquema de aproximação completa (Full Approximation Scheme, FAS). De acordo com Briggs et al. (2000) e Trottenberg et al. (2001), o esquema CS é geralmente aplicado a problemas lineares e o FAS, a problemas não-lineares.

Na Figura 1 pode-se observar a ilustração para uma malha estruturada, onde estão descritos os cálculos realizados em cada nível de malha. Ao lado do ciclo, são mostradas as malhas utilizadas para um ciclo $\mathrm{V}$, iniciando com uma malha de 33×33 elementos até uma malha de $3 \times 3$ (a malha mais grossa). Esta forma de se visitar as malhas, que é utilizada para o método multigrid geométrico, pode ser aplicada de maneira análoga ao AMG.

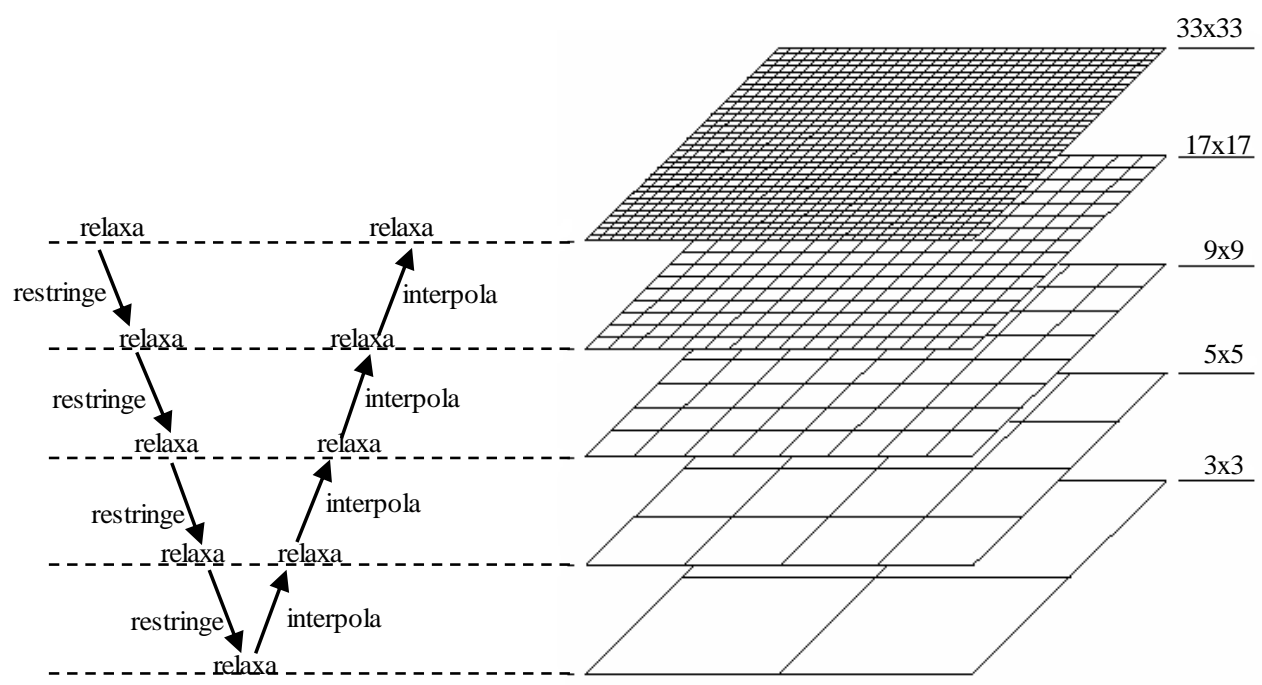

Figura 1. Ciclo V (Adaptado de http://www.mgnet.org/mgnet/tutorials/xwb/mg.html) 
Outros tipos de ciclos (W, F, entre outros) podem ser obtidos por analogia ao método multigrid geométrico.

Diversos trabalhos utilizando o método multigrid são encontrados na literatura e a escolha dos componentes multigrid têm um papel fundamental na convergência ou não deste método. Entende-se por componentes do método multigrid: ciclo (ordem com que as diferentes malhas são visitadas), número de iterações internas (vezes em que o problema na malha fina é resolvido), número de níveis (quantidade de malhas auxiliares empregadas na resolução do problema com o emprego do método multigrid). A escolha dos componentes, muitas vezes, torna-se difícil e uma pequena alteração no método pode melhorar sensivelmente seu desempenho. De acordo com Trottenberg et al. (2001), uma simples modificação no algoritmo resulta em uma redução significante no tempo de processamento requerido. De acordo com Langer e Pusch (2006), a eficiência do método multigrid está relacionado à adaptação das componentes do método multigrid, que também são feitas de acordo com o delineamento físico e a formulação variacional do problema.

\subsection{Descrição da representação computacional da propagação de ondas oceânicas em tanques}

\subsubsection{Domínio computacional}

Uma possibilidade para analisar numericamente fenômenos relacionados à propagação e ondas oceânicas é através de tanques numéricos de ondas, conforme o indicado esquematicamente na Figura 2. No presente trabalho, é considerada uma onda regular com características constantes, com período $(T)$ igual a $5 \mathrm{~s}$ e comprimento $(\lambda)$ igual a $37,5 \mathrm{~m}$.

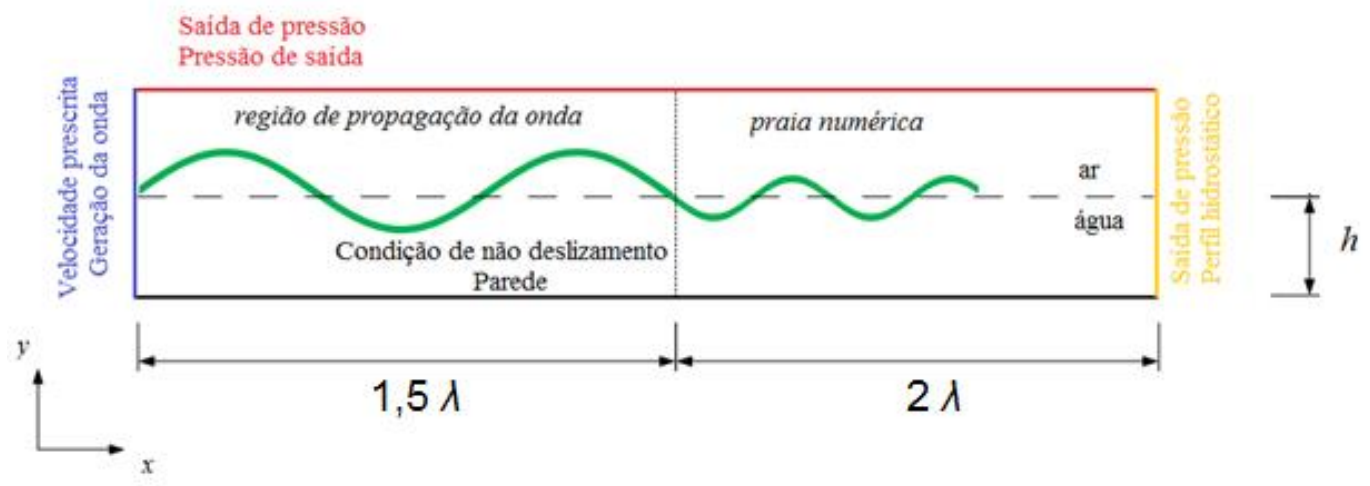

Figura 2. Representação esquemática de um tanque de ondas 
Para representar o fenômeno de forma adequada é necessário que o dimensionamento do tanque de ondas esteja em harmonia com as características da onda a ser analisada. Conforme é indicado na Figura 2 o tanque de ondas é dividido em duas regiões: 1) região da propagação da onda e 2) região de absorção da onda (praia numérica) - região onde a reflexão da onda é eliminada.

\subsubsection{Condições de contorno e condição inicial}

Num tanque de ondas, a principal condição de contorno diz respeito ao gerador da onda desejada. Como se observa na Figura 2, através de uma condição de velocidade prescrita, o gerador de ondas é posicionado do lado esquerdo do tanque. Numericamente, para a geração de ondas consideram-se as componentes horizontais $(u)$ e verticais $(v)$ da onda que são dadas por (DEAN \& DALRYMPLE, 1991; CHAKRABARTI, 2005):

$$
\begin{aligned}
& u=\frac{H}{2} g k \frac{\cosh (k z+k h)}{\omega \cosh (k h)} \cos (k x-\omega t)+\frac{3}{4}\left(\frac{H}{2}\right)^{2} \omega k \frac{\cosh 2 k(k+z)}{\sinh ^{4}(k h)} \cos 2(k x-\omega t) \\
& v=\frac{H}{2} g k \frac{\operatorname{senh}(k z+k h)}{\omega \cosh (k h)} \operatorname{sen}(k x-\omega t)+\frac{3}{4}\left(\frac{H}{2}\right)^{2} \omega k \frac{\operatorname{senh} 2 k(k+z)}{\operatorname{senh}^{4}(k h)} \operatorname{sen} 2(k x-\omega t)
\end{aligned}
$$

em que, $H$ é a altura da onda incidente $(\mathrm{m}) ; g$ é a aceleração da gravidade $\left(\mathrm{m} / \mathrm{s}^{2}\right) ; k$ é o número de ondas, $h$ é a profundidade $(m), \omega$ é a frequência, $x$ é a coordenada que representa a direção principal (m), $t$ é o tempo (s) e $z$ é a coordenada normal (m).

$\mathrm{Na}$ linha da direita (linha laranja na Figura 2), é adotado um perfil hidrostático como condição de contorno. Entre o dispositivo e essa linha está inserida uma praia numérica, que tem como objetivo eliminar a reflexão das ondas dentro do tanque. A utilização do método da praia numérica consiste em adicionar termos de sorvedouro nas equações de quantidade de movimento, em uma dada região do tanque. Esses termos são dados por (LISBOA et al., 2016; DIZADJI \& SAJADIAN, 2011):

$$
S=-\left[C_{1} \rho V+\frac{1}{2} C_{2} \rho|V| V\right]\left(1-\frac{z-z_{f s}}{z_{b}-z_{f s}}\right)
$$

onde $C_{1}$ e $C_{2}$ são os coeficientes de amortecimento linear e quadrático, respectivamente; $\rho$ é a massa específica do fluido; $V$ é a velocidade; $z$ é a posição normal; $z_{f s}$ é a posição 
vertical da superfície livre, $z_{b}$ a posição vertical do fundo; $x$ representa a posição horizontal, enquanto que $x_{s}$ marca o início e $x_{e}$ o fim da praia numérica, e $S$ é o momento de afundamento. Seguindo as recomendações de Lisboa et al. (2016), $C_{1}=20$ e $C_{2}=0$.

Na superfície lateral esquerda superior, assim como na parte superior do tanque é aplicada uma condição de contorno de pressão atmosférica. Já na parede inferior do tanque de ondas, é imposta a condição de não deslizamento e impermeabilidade com velocidade nula.

Como condição inicial, foi imposto o fluído em repouso (condição flat), com profundidade $h=10 \mathrm{~m}$ e $t=0$ na equação da elevação da superfície livre que segue.

$$
\eta(x, t)=A \cos (k x-\omega t)+\frac{A^{2} k \cosh (k h)}{4 \operatorname{senh}^{3}(k h)}[2+\cosh (2 k h) \cos 2(k x-\omega t)]
$$

na qual: $A$ é a amplitude da onda $(\mathrm{m})$, dado por $H / 2$, x é a posição $(\mathrm{m}), t$ é a variação do tempo (s), $k$ e $\omega$ são o número de onda $\left(\mathrm{m}^{-1}\right)$ e a frequência da onda ( $\mathrm{rad} / \mathrm{s}$ ), respectivamente.

\subsubsection{Discretização espacial}

A discretização espacial neste trabalho é igual em todos os casos analisados e foi construída através da metodologia de malha stretched. Essa estratégia adota a definição de regiões mais refinadas que outras, sendo estas regiões de interesse da investigação como a superfície livre, como apresentado na Figura 3 (MAVRIPILIS, 1997).

Conforme pode ser observado na Figura 3, e até mesmo considerando a Figura2, o domínio computacional é dividido em seis regiões de interesse para geração da malha refinada em diferentes partes. A malha empregada neste trabalho tem um total de 15.000 volumes, sendo que o detalhamento do número de volumes por direção pode ser verificado na Tabela 1. Por exemplo, considerando a Região 2, onde efetivamente ocorre a propagação da onda, pode-se observar na Tabela 1 uma discretização horizontal de 75 volumes (o que garante uma discretização de 50 volumes por comprimento de onda) e de 40 volumes na vertical (o que garante uma discretização de 20 volumes por altura de onda, conforme o indicado em Gomes (2014)). 


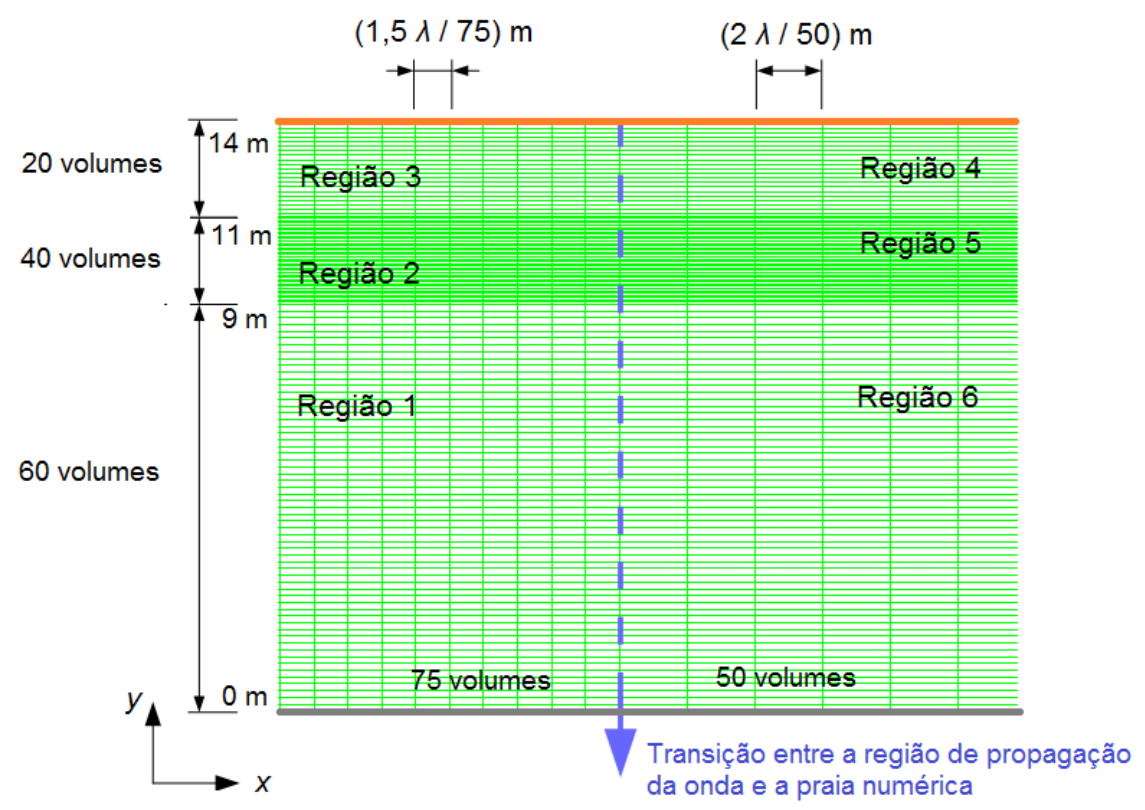

Figura 3. Detalhes da discretização espacial

Tabela 1. Discretização espacial por regiões.

\begin{tabular}{ccccccc}
\hline & \multicolumn{6}{c}{ Regiões } \\
\cline { 2 - 7 } Direção & \multicolumn{7}{c}{ Número de volumes em cada direção } \\
\cline { 2 - 7 } & $\mathbf{1}$ & $\mathbf{2}$ & $\mathbf{3}$ & $\mathbf{4}$ & $\mathbf{5}$ & $\mathbf{6}$ \\
\hline Horizontal & 75 & 75 & 75 & 50 & 50 & 50 \\
\hline Vertical & 60 & 40 & 20 & 60 & 40 & 20 \\
\hline
\end{tabular}

\subsection{Modelo Matemático e Numérico}

Nesse trabalho é empregado o método Volume of Fluid VOF (HIRT e NICHOLS, 1981). O método VOF é um modelo multifásico usado para a solução de escoamentos compostos de dois ou mais fluidos imiscíveis. Nesta formulação, todas as fases são definidas e o volume ocupado por uma fase não pode ser ocupado por outra fase. Assim, para representar as fases contidas em cada volume de controle, é necessário o conceito da fração de volume $(\alpha)$. Consequentemente, é necessário que a soma de todas as fases em cada célula seja sempre igual a um. No modelo numérico apresentado neste trabalho, somente duas fases são consideradas: a água e o ar. Portanto, as células com valores de

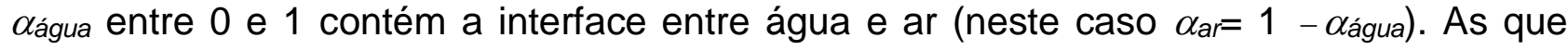
possuem $\alpha_{\text {água }}=0$ estão sem água e completas de $\operatorname{ar}\left(\alpha_{a r}=1\right)$; e, por sua vez, as que apresentam $\alpha_{a r}=0$ estão cheias de água ( $\alpha_{\text {água }}=1$ ). 
No modelo VOF, as equações de conservação da massa, momento e transporte de fração volumétrica são empregadas quando há dois fluidos imiscíveis, ar e água neste caso. Para resolvê-las, utiliza-se o Método dos Volumes Finitos (VERSTEEG \& MALALASEKERA, 2007). A equação da conservação da massa é dada por:

$$
\frac{\partial \rho}{\partial t}+\nabla \cdot(\rho \cdot \vec{v})=0
$$

onde $\rho$ é a densidade da mistura $\left(\mathrm{kg} / \mathrm{m}^{3}\right)$ e $\vec{v}$ é o vetor velocidade do fluxo (m/s).A equação da conservação do momento é:

$$
\frac{\partial(\rho \vec{v})}{\partial t}+\nabla \cdot(\rho \vec{v} \vec{v})=-\nabla p+\nabla(\tau)+\rho g
$$

onde p é a pressão (Pa) e $\tau$ é o tensor de deformação $\left(\mathrm{N} / \mathrm{m}^{2}\right)$.

Neste estudo, as frações de volume $\alpha$ representam as fases dentro do problema. Se a célula possui água, $\alpha_{\text {água }}=1$ e $\alpha_{a r}=1-\alpha_{\text {água }}$. Assim, a equação para as frações de volumes é

$$
\frac{\partial(\rho \alpha)}{\partial t}+\nabla \cdot(\rho \cdot \alpha \vec{v})=0
$$

As densidades e viscosidade da mistura são dadas por:

$$
\begin{aligned}
& \rho=\alpha_{\text {água }} \rho_{\text {água }}+\alpha_{a r} \rho_{a r} \\
& \mu=\alpha_{\text {água }} \mu_{\text {água }}+\alpha_{a r} \mu_{a r}
\end{aligned}
$$

Para a solução numérica dessas equações utilizou-se o método PRESTO para discretizar o momento e a pressão. Para o acoplamento pressão-velocidade, foi empregado o método PISO, enquanto que o GEO-RECONSTRUCTION foi utilizado na fração volumétrica.

Todas a simulações foram realizadas usando um computador com processador AMD A6-5400B APU de 3.60 GHz e 8 GB de memória RAM. Além disso, todas as simulações foram rodadas em série. 


\section{RESULTADOS}

\subsection{ANÁLISE DO NÚMERO DE PRÉ E PÓS SUAVIZAÇÃO}

Os testes computacionais foram resolvidos com o software FLUENT, onde é empregado o ciclo $\mathrm{V}$ para o escoamento e ciclo flexível para a turbulência. Dentro do ciclo V, podem ser escolhidos o número de pré e pós suavizações (número de vezes que o sistema de equações vai ser resolvido a cada malha visitada). Conforme os trabalhos de Oliveira et al. (2018), Santiago et al. (2015) e Suero et al. (2012), o valor ótimo para o número de iterações internas é diferente de 1, valor que é fortemente recomendado pela literatura (RUGE e STÜBEN, 1986; KRECHEL e STÜBEN, 1999; BRIGGS et al. 2000; IWAMURA et al. 2003; FALGOUT, 2006 e TROTTENBERG et al. 2001). Além disso, número de pré e pós suavizações igual a 1 é o valor padrão empregado no algoritmo implementado no FLUENT.

Conforme pode ser observado na Figura 4, o valor ótimo para o parâmetro em questão é igual a 2. Pode-se observar ainda que o tempo computacional para a resolução do problema é reduzido praticamente à metade, uma diminuição de cerca de $47 \%$ do tempo de processamento, o equivalente a $9 \mathrm{~h}$. Além disso, observa-se que, o valor considerado padrão para o FLUENT apresenta o maior tempo computacional dentre todos os que foram testados.

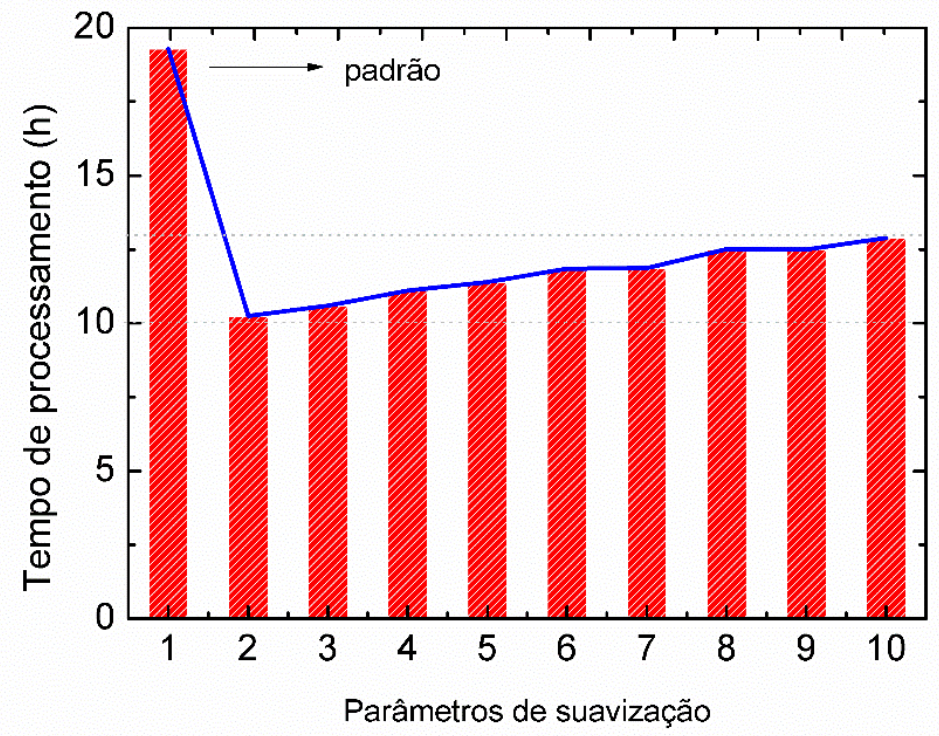

Figura 4. Tempo de processamento em relação aos parâmetros de suavização 


\subsection{ANÁLISE DO NÚMERO DE NÍVEIS}

Com o número de pré e pós suavizações determinado pelos testes descritos na seção anterior, ou seja, iguais a 2, foram realizados testes computacionais para verificar a influência do número de níveis de malha no tempo de processamento.

Na Figura 5 é possível observar que o número de níveis empregado para a resolução do problema via método multigrid, também influencia o tempo computacional. $O$ valor ótimo para este parâmetro é 45 enquanto o valor padrão para o software FLUENT é 40, isso significa uma diminuição de cerca de $1 \%$ no tempo de processamento em relação ao padrão, cerca de 0,10 h. Diversos autores como Ruge e Stüben (1986), Falgout (2006) e Langer e Pusch (2006), em seus trabalhos, não se preocupam com o número de malhas a ser empregado. Nos trabalhos em que este dado está explícito, observa-se a utilização de todos os níveis de malha, como por exemplo em Krechel e Stüben (1999), Wu e Elman (2006) e Iwamura et al. (2003). Suero et al. (2012), Santiago et al. (2015) e Oliveira et al. (2018), testaram e concluíram que o número ótimo de níveis é igual ao número máximo de níveis que pode ser empregado.

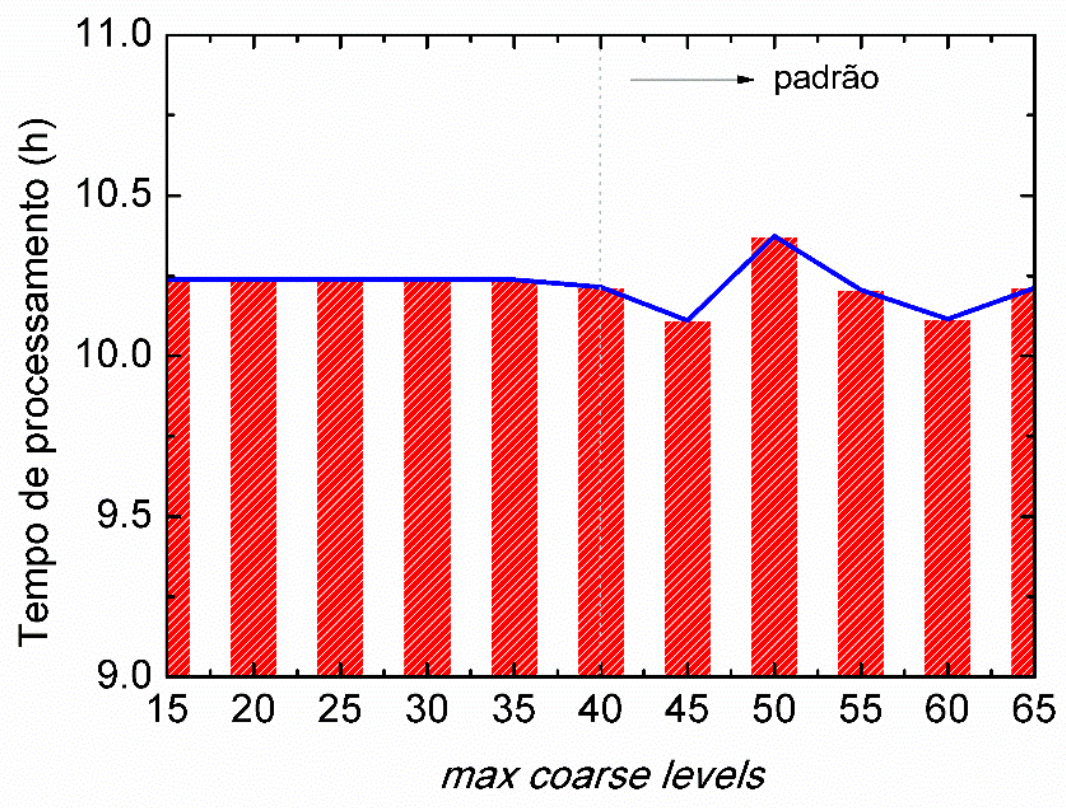

Figura 5. Tempo de processamento em relação ao número de níveis de malha 


\subsection{ANÁLISE DO NÚMERO DE PASSOS DE SUAVIZAÇÃO}

Outra análise é em relação ao número de vezes que o método de suavização é aplicado cada vez que um relaxamento é realizado. O método escolhido é o Método de Gauss-Seidel.

Na Figura 6 observa-se que o número ótimo de passos de suavização é igual a 1, sendo diferente do valor padrão empregado pelo FLUENT, que é igual a 2. Este resultado concorda com Ruge e Stüben (1986), Krechel e Stüben (1999), Briggs et al. (2000), Trottenberg et al. (2001), Iwamura et al. (2003) e Falgout (2006), que colocam que o número de passos de suavização deve ser igual a 1.

É possível verificar na Figura 6 que a diferença entre o caso ótimo e o pior caso chega a cerca de $2,5 \mathrm{~h}$.

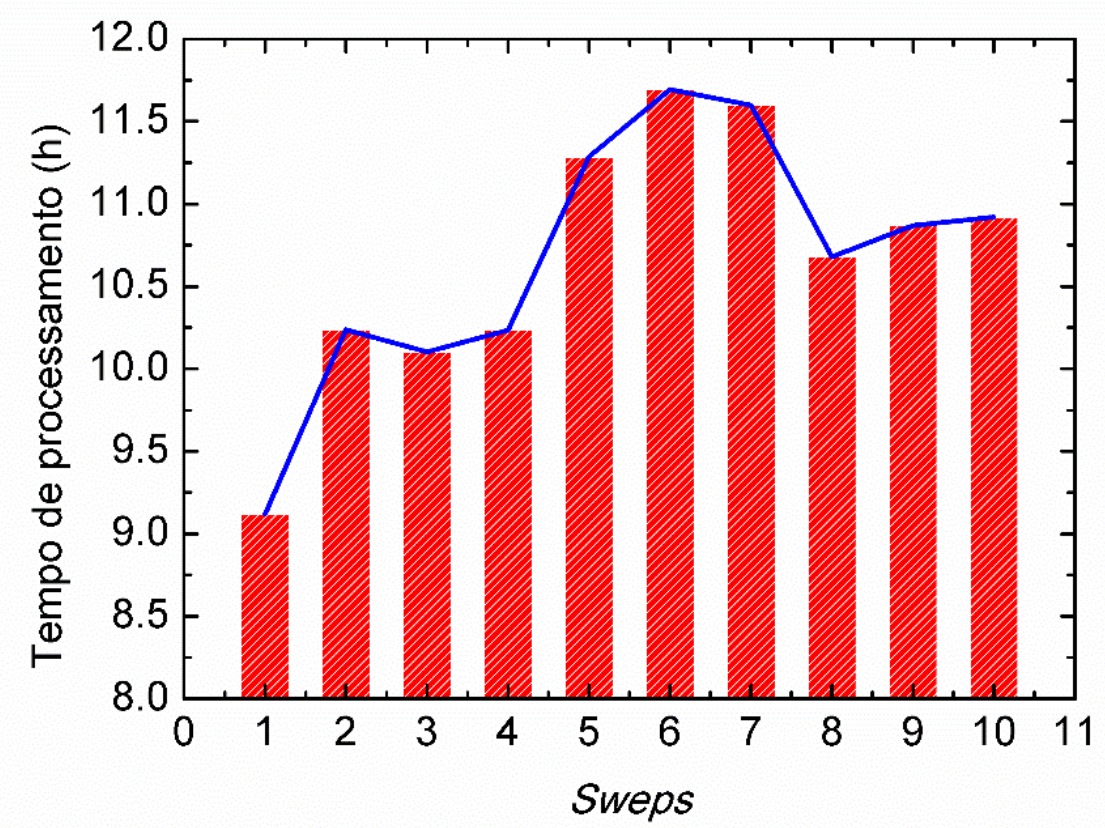

Figura 6. Tempo de processamento em relação ao número de passos de suavizações

\section{CONSIDERACQOESS FINAIS}

No presente trabalho foi realizada uma análise do emprego do método multigrid algébrico nas simulações numéricas, que envolvem a propagação de ondas oceânicas em tanques. O problema em questão envolve a simulação da propagação de uma onda com período de $5 \mathrm{~s}$ e comprimento de $37,5 \mathrm{~m}$. O domínio computacional é bidimensional e representado por um tanque de ondas numérico dividido em uma região de propagação e outra de absorção da onda. Foram testados diferentes valores para o número de iterações 
internas e o número de níveis. Para o número de iterações internas, o valor ótimo encontrado foi igual a 2, que difere do valor fortemente recomendado pela literatura, que é igual a 1, mesmo valor default do FLUENT. O número de níveis ótimo também difere do valor empregado por default no software: 45 é o valor ótimo, enquanto que 40 é o valor padrão. Os resultados obtidos após as otimizações, conduziram a uma redução de tempo de $10,16 \mathrm{~h}$.

No entanto, reforça-se que se faz necessária a realização de testes computacionais tendo como objetivo encontrar os parâmetros ótimos para cada problema a ser resolvido. Conforme Langer e Pusch (2006), a eficiência do método multigrid está relacionado à adaptação das componentes do método multigrid, que também são feitas de acordo com o delineamento físico e a formulação variacional do problema.

\section{AGRADECIMENTOS}

Os autores agradecem ao Instituto Federal do Paraná (IFPR), à Universidade Federal do Rio Grande (FURG), à Universidade do Vale do Rio dos Sinos (Unisinos), à Fundação Araucária, à Fundação de Amparo à Pesquisa do estado do Rio Grande do Sul (FAPERGS) e ao Conselho Nacional de Desenvolvimento Cientifico e Tecnológico (CNPq).

\section{REFERENCIAS}

BRIGGS, W. L. et al. A Multigrid Tutorial. $2^{2}$ ed. SIAM, 2000.

CARNEIRO, M. L. Desenvolvimento de dispositivo de geração e absorção ativa de ondas para tanque de ensaio de estruturas oceânicas. Dissertação (Mestrado, Escola Politécnica da Universidade de São Paulo, São Paulo - SP, 2007.

CHAKRABARTI, S. K. Handbook of offshore engineering. Elsevier, Ilinois, Estados Unidos, 2005.

CRAIG, C. D._12122_tex2html_wrap26.gif. 1995. Altura: 587 pixels. Largura: 372 pixels. $6.15 \mathrm{~Kb}$. Formato GIF Image.

Disponível em: < http://www.mgnet.org/mgnet/tutorials/xwb/mg.html >. Acesso em 20 set. 2007.

DEAN, R. G., DALRYMPLE, R.A. Water wave mechanics for engineers and scientists. World Scientific, 2, Singapura, 1991.

DIZADJI, N.; SAJADIAN, S. E. Modeling and optimization of the chamber of OWC system. Energy, 36, p. 2360-2366, 2011. 
FALGOUT, R. D. An Introduction to Algebraic Multigrid. Computing in Science and Engineering, p. 1-11, 2006.

GOMES, M. N. et al. Computational modeling of a regular wave tank. Energia Térmica, 8, p. 44-50, 2009.

GOMES, M, N. Constructal Design de Dispositivos Conversores de Energia das Ondas do Mar em Energia Elétrica do Tipo Coluna de Água Oscilante. Tese de Doutorado, PROMEC/UFRGS, Porto Alegre, 2014.

GUPTA, M. M. et al. A compact multigrid solver for convection-diffusion equations. Journal of Computational Physics, vol. 132, p. 123-129, 1997.

HIRT, C.W.; NICHOLS, B.D. Volume of fluid (VOF) method for the dynamics of free boundaries. Journal of Computational Physics, 39(1), p. 201-225, 1981.

IWAMURA, C. et al. An efficient algebraic multigrid preconditioned conjugate gradient solver. Computer Methods in Applied Mechanicsand Engineering, vol. 192, p. 2299-2318, 2003.

KRECHEL, A. \& STÜBEN, K. Operator Dependent Interpolation in Algebraic Multigrid. Proceedings of Fifth European Multigrid Conference, 24 p, 1999.

LANGER, U. \& PUSCH, D. Comparison of Geometrical and Algebraic Multigrid Preconditioners for Data-Sparse Boundary Element Matrices. Springer-Verlag Berlin Heidelberg, p. 130-137, 2006.

LISBOA, R. C. et al. Regular and Irregular Wave Propagation Analysis in a Flume with Numerical Beach Using a Navier-Stokes Based Model. Defect and Diffusion Forum, 327, p. 81-90, 2016.

MALISKA, C. R. Transferência de Calor e Mecânica dos Fluidos Computacional. Livros Técnicos e Científicos, Editora S.A., Rio de Janeiro, 2004.

MARTINS, J. C. et al. Geometric evaluation of the main operational principle of na overtopping wave energy converter by means of Constructal Design. Renewable Energy, v. 118, p. 727-741, 2018.

MAVRIPILIS, D. J. Unstructured Drid Techniques. Annual Reviews Fluid Mechanics, 29, p. 473-514, 1997.

OLIVEIRA, F DE. et al. The effect of multigrid parameters in a $3 \mathbf{d}$ heat diffusion equation. Int. J. of Applied Mechanics and Engineering, vol. 23, nำ1, pp. 213-221, 2018.

RUGE, J. \& STÜBEN, K. Algebraic Multigrid (AMG). S. F. McCormick, Multigrid Methods, volume 5 de Frontiers in Applied Mathematics, SIAM, 1986.

RUTZ, G. V., PINTO, M. A. V. Análise de Fourier local e otimização de parâmetros do método multigrid para a equação de difusão anisotrópica. In: CILAMCE - XXXVII Iberian Latin American Congress in Computational Methods in Engineering, 2016. 
SANTIAGO, C. D. et al. Performance of geometric multigrid for coupled twodimensional systems in CFD. Applied Mathematical Modelling, vol. 39, p. 2602-2615, 2015.

SUERO, R. et al. Analysis of algebraic multigrid parameters for two-dimensional steady-state heat diffusion equation. Applied Mathematical Modeling, vol. 36, p. 29963006, 2012.

STÜBEN, K. A review of algebraic multigrid. Journal of Computational and Applied Mathematics, vol. 128, p. 281-309, 2001.

TROTTENBERG, U. et al. Multigrid. Academic Press, 2001.

VERSTEEG, H. K.; MALALASEKERA, W. An Introduction to Computational Fluid Dynamics - The Finite Volume Method. Pearson, England, 2007.

WESSELING, P. An Introduction to Multigrid Methods. John Wiley \& Sons, 1992.

WU, C. T., ELMAN, H. C. Analysis and comparison of geometric and algebraic multigrid for convection-diffusion equations. SIAM Journal on Scientific Computing, v. 28, n 6, p. 2208-2228, 2006. 